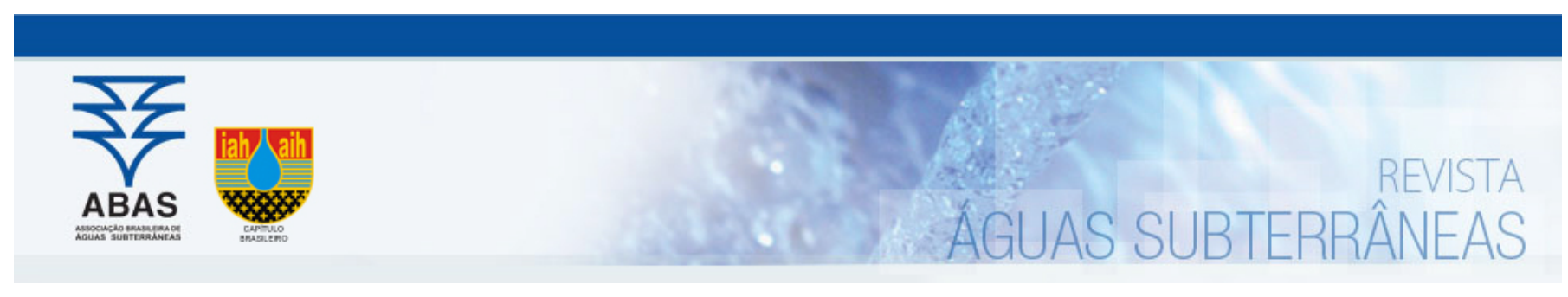

Artigos

\title{
Análise hidroquimíca e da aplicação de diferentes métodos de avaliação da qualidade da água subterrânea em aquíferos costeiros em Osório - RS
}

\section{Hidrochemical analysis and the application of different groundwater quality assessment methods in coastal aquiferos in Osorio - RS}

\section{Rosana Alves Paim¹; Pedro Antonio Roehe Reginato2; Elias Zientarski Michalski3; Rosane Maria Lanzer³ Tuane de Oliveira Dutra4}

1 Programa de Pós-Graduação em Recursos Hídricos e Saneamento Ambiental Instituto de Pesquisas Hidráulicas - IPH, Porto Alegre, RS 2 Departamento de Hidromecânica e Hidrologia / Instituto de Pesquisas Hidráulicas - IPH / Universidade Federal do Rio Grande do Sul UFRGS, Porto Alegre, RS

3 Universidade de Caxias do Sul (UCS), Caxias do Sul, RS

4 Programa de Pós-Graduação em Recursos Hídricos e Saneamento Ambiental / Instituto de Pesquisas Hidráulicas - IPH / Universidade

Federal do Rio Grande do Sul, Porto Alegre, Rio Grande do Sul, Brasil

\rosana.a.paim@gmail.com,pedro.reginato@ufrgs.br,ezmichal@ucs.br, rlanzer@ucs.br, tuanehidrica@gmail.com

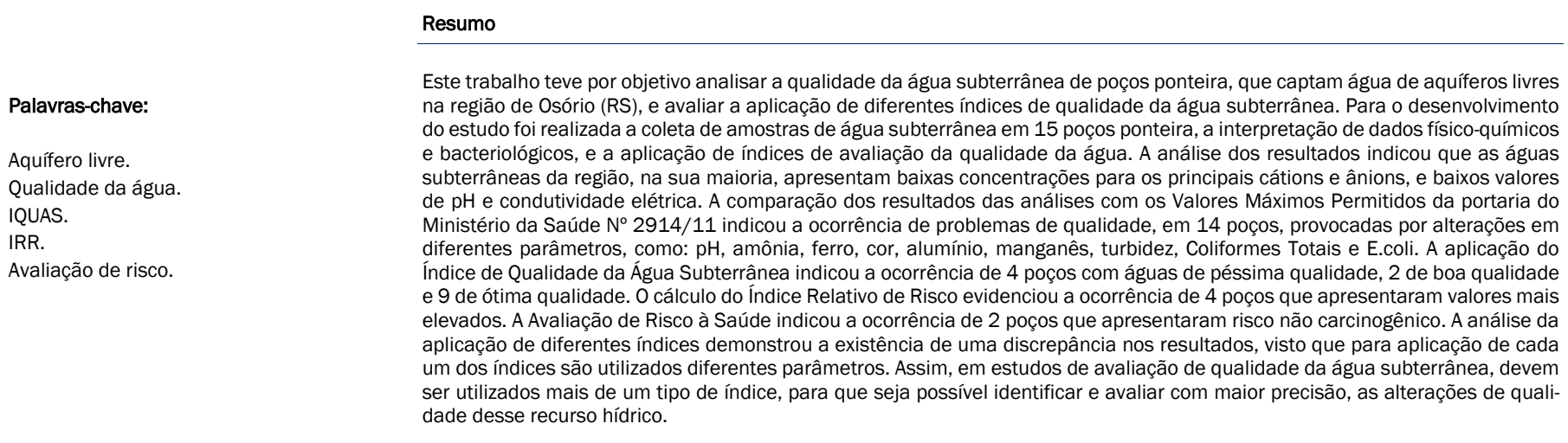

\section{Abstract}

Keywords:

Unconfined aquifer. Water quality.

IQUAS.

IRR.

Risk assessment.
This work aimed to analyze the groundwater quality of driven-point wells, which capture water from unconfined aquifers in the Osório region (RS), and to evaluate the application of different groundwater quality indices. For the development of the study, collection of groundwater samples in 15 driven-point wells, interpretation of physical-chemical and bacteriological data, and application of water quality were carried. The evaluation of the results indicated that the groundwater of the region mostly presents low concentrations for the main cations and anions, low values of $\mathrm{pH}$ and electrical conductivity. The comparison of the results of the analyzes with the Maximum Allowable Values of Ministry of Health No. 2914/11 indicated the existence of quality problems in 14 wells caused by changes in different parameters, such as $\mathrm{pH}$, ammonia, iron, color, aluminum, manganese, turbidity, total coliforms, and E. coli. The application of the Groundwater Quality Index indicated the occurrence of 4 wells with water of poor quality, 2 of good quality and 9 of high quality. The calculation of the Relative Risk Index evidenced the occurrence of 4 wells which presented higher values. The Health Risk Assessment indicated the occurrence of 2 wells that presented non-Carcinogenic Risk. The analysis of the application of different indices demonstrated the existence of a discrepancy in the results, since the application of each of the indices different parameters are used. Thus, in groundwater quality assessment studies, more than one type indices should be used, so that it is possible to identify and evaluate more precisely the changes in the quality of this water resource. 


\section{INTRODUÇÃO}

A avaliação da qualidade da água subterrânea pode ser realizada por meio da análise de diferentes parâmetros físico-químicos e bacteriológicos, bem como pela aplicação de diferentes índices. Como essa avaliação envolve a análise de muitos dados e variáveis, uma solução pode ser dada, pelo tratamento multivariado dos dados (Franca et al., 2018).

Uma forma de avaliar a qualidade da água é a partir da aplicação de Índices de Qualidade da Água (IQA), que são ferramentas matemáticas utilizadas para transformar vários parâmetros em uma única grandeza, que represente o nível de qualidade da água. O uso de um IQA é prático, sendo uma diretriz de condução, pois os programas de acompanhamento de qualidade da água, ao longo do tempo, geram um grande número de dados analíticos que precisam ser apresentados num formato sintético, para que representem e descrevam de forma compreensivel e significativa o estado e as tendências da qualidade da água (Ferreira e Ide, 2001; Sánchez et al., 2007).

De acordo com Almeida (2012) e Santos (2009), o desenvolvimento dos índices de avaliação da qualidade de água surgiu nos anos 60 com o objetivo de promover uma avaliação geral da qualidade da água ou de avaliar a qualidade das águas superficiais. Com o passar dos anos foram desenvolvidos diferentes índices, sendo muitos deles voltados para a avaliação da qualidade da água subterrânea. Os principais índices desenvolvidos e aplicados na avaliação da água subterrânea são apresentados na Tabela 1.

Tabela 1 - Principais índices de avaliação da qualidade da água subterrânea

\begin{tabular}{|c|c|c|}
\hline Índice & Objetivo/Parâmetros & Referência \\
\hline SEQ & $\begin{array}{l}\text { Representa as particularidades da água subterrânea. Avalia grupos de alteração } \\
\text { (sabor, odor; matéria orgânica, partículas em suspensão, ferro, manganês, cor, mi- } \\
\text { croorganismos, mineralização e salinidade, nitrato, micropoluentes minerais, pes- } \\
\text { ticidas, hidrocarbonetos, micropoluentes orgânicos, corrosão, formação de depósi- } \\
\text { tos, temperatura) de acordo com o uso da água. }\end{array}$ & $\begin{array}{l}\text { Cadilhac e Albinet } \\
\text { (2003) }\end{array}$ \\
\hline IQNAS & $\begin{array}{l}\text { Avaliação da Qualidade da Água Subterrânea de Aquíferos do estado da Bahia. Ba- } \\
\text { seado na análise de parâmetros como pH, cloreto, resíduos totais, dureza, nitrato, } \\
\text { flúor. }\end{array}$ & Oliveira et al., 2007 \\
\hline IQUAS & $\begin{array}{l}\text { Elaborado com base nos índices SEQ e IQNAS para avaliação geral da qualidade } \\
\text { das águas subterrâneas. Baseado em grupos de alteração (partículas em suspen- } \\
\text { são, ferro e manganês, microorganismos, mineralização e salinidade, nitratos, ni- } \\
\text { trogenados (excluindo-se nitrato), micropoluentes minerais e os micropoluentes or- } \\
\text { gânicos }\end{array}$ & $\begin{array}{l}\text { Almeida e Oliveira } \\
\text { (2008) }\end{array}$ \\
\hline IQASCH & $\begin{array}{l}\text { Avaliação da qualidade da água para consumo humano em áreas com atividades } \\
\text { agrícolas. Baseado na análise de: condutividade elétrica, dureza, pH, turbidez, ni- } \\
\text { trato, coliformes termotolerantes, ferro, manganês, arsênio, bário, fluoreto e pesti- } \\
\text { cidas. }\end{array}$ & Santos (2009) \\
\hline AFC & $\begin{array}{l}\text { Método desenvolvido para avaliar o impacto da agricultura na qualidade da água } \\
\text { subterrânea. Utiliza parâmetros como nitrato, sulfato, cloreto e cálcio, mas permite } \\
\text { o uso de outros parâmetros. }\end{array}$ & $\begin{array}{l}\text { Stigter et al., } \\
(2006)\end{array}$ \\
\hline IRQ & $\begin{array}{l}\text { Desenvolvido para caracterizar e hierarquizar o potencial qualitativo da água sub- } \\
\text { terrânea. Utiliza os parâmetros nitrato, cloreto e sólidos totais dissolvidos. No en- } \\
\text { tanto, outros parâmetros podem ser utilizados. }\end{array}$ & $\begin{array}{l}\text { Fernando e Lou- } \\
\text { reiro }(2006)\end{array}$ \\
\hline e-IQUAS & $\begin{array}{l}\text { Método que permite a seleção de parâmetros, que devem ser agregados em "Gru- } \\
\text { pos de Alteração", para aplicação do índice. São propostos oito grupos de alteração } \\
\text { que são: agrotóxicos e pesticidas; elementos filtráveis e partículas; matéria orgâ- } \\
\text { nica e nutrientes; micro-organismos, micropoluentes minerais, micropoluentes or- } \\
\text { gânicos, mineralização e salinidade, PCB. }\end{array}$ & $\begin{array}{l}\text { Almeida e Oliveira } \\
(2017)\end{array}$ \\
\hline
\end{tabular}

Segundo Batista e Cabral (2017), a escolha de um determinado índice deve levar em consideração os objetivos do estudo da avaliação que está sendo realizada, pois cada índice utiliza diferentes parâmetros. Para Coutinho et al. (2013), a escolha dos parâmetros que devem ser utilizados nos cálculos dos índices deve estar embasada no uso da água, pois dependendo do uso, haverá maior ou menor restrição a qualidade da água.

Na região abrangida pelo município de Osório (RS), a água subterrânea consiste num recurso de grande importância, que está associado ao Sistema Aquífero Quaternário Costeiro (Machado e Freitas, 2005). As águas subterrâneas desse sistema aquífero são utiliza- das no abastecimento público e doméstico (área urbana e rural), na dessedentação de animais, na irrigação e no desenvolvimento de atividades industriais e comerciais. Em geral, a captação é realizada por poços ponteira, que possuem pequena profundidade e captam água de aquíferos granulares livres, que ocorrem em camadas de sedimentos arenosos (REGINATO et al., 2017).

Para essa região e sistema aquífero não há trabalhos de aplicação de índices de avaliação da qualidade da água subterrânea. Há estudos que envolvem a análise hidroquímica e a avaliação da qualidade da água (baseados principalmente na comparação de resulta- 
dos com valores máximos permitidos em portarias de potabilidade). Um exemplo é o trabalho realizado por Reginato et al. (2017), desenvolvido para o município de Osório. Nesse trabalho, os autores identificaram a ocorrência de problemas de qualidade, que ocorrem associados a presença de ferro, manganês e fluoreto, bem como a alterações nos parâmetros cor e turbidez.

Em função da importância da água subterrânea para a região foi desenvolvido um estudo que teve como objetivo a análise das características hidroquímicas e da qualidade da água subterrânea que é utilizada para abastecimento. Essa análise foi realizada a partir da aplicação de índices de qualidade da água e avaliação de risco a saúde humana, bem como pela comparação com os Valores Máximos Permitidos (VMP), disponíveis em portarias de potabilidade, como a N²914/11, do Ministério da Saúde (Brasil, 2011). Com os resultados obtidos foi possível analisar a qualidade da água subterrânea, bem como avaliar o uso dos diferentes métodos.

\section{MATERIAIS E MÉTODOS}

\subsection{Caracterização Hidrogeológica}

A caracterização hidrogeológica foi realizada por meio da análise de dados geológicos e hidrogeológicos de poços que foram identificados e cadastrados na região de estudo. Os perfis geológicos e construtivos dos poços cadastrados foram utilizados para identificar as principais unidades aquíferas que formam o Sistema Aquífero Quaternário Costeiro e que são captadas por esses poços.

\subsection{Caracterização Hidroquímica}

Para realização da caracterização hidroquímica foram selecionados poços, tendo como critérios o uso para abastecimento doméstico ou coletivo (escolas), o tipo de captação (poço ponteira ou escavado) e a existência de dados como a profundidade dos poços. Ao todo, foram selecionados 15 pontos de captação, sendo 12 poços ponteira e 3 poços cacimba (Figura 1).

Figura 1 - Mapa de localização dos poços onde foram coletadas amostras e realizadas análises
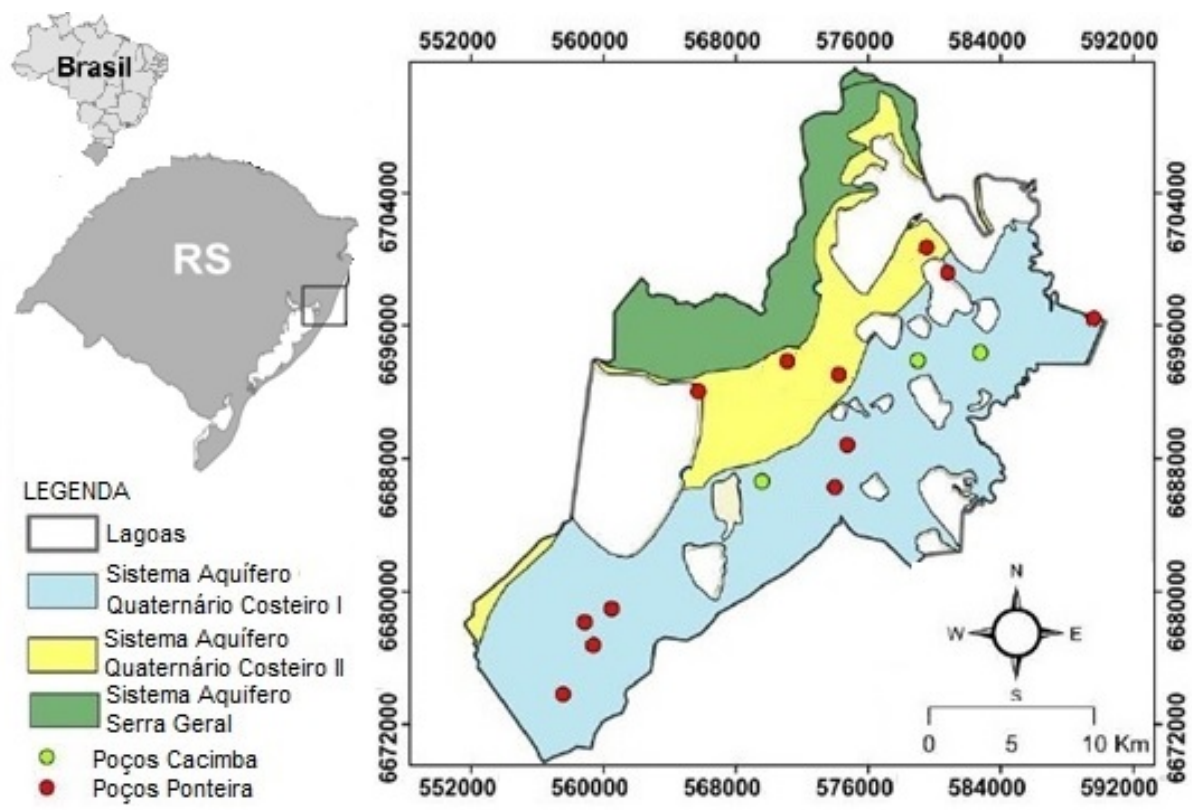

Em cada poço foram realizadas medições em campo dos parâmetros pH e Condutividade Elétrica (CE), com o emprego de sonda multiparâmetros EUTECH (modelo PCTestr 35), bem como a coleta das amostras. A coleta de amostras de água foi feita na saída da boca do poço ou após a bomba, sendo que poços parados tiveram as bombas acionadas, sendo a coleta realizada após 10 minutos de bombeamento. As amostras foram acondicionados em frascos esterilizados, fornecidos pelo Laboratório de Análises Físico-Químicas do Centro de Ecologia da UFRGS. Nesse laboratório, foram analisados os seguintes parâmetros: Alcalinidade, Cloretos $(\mathrm{Cl})$, Cor, Alumínio (Al), Bário (Ba), Cádmio (Cd), Cálcio (Ca), Cobalto (Co), Cobre $(\mathrm{Cu})$, Chumbo (Pb), Cromo (Cr), Dureza, Ferro (Fe), Fluoreto (F), Lítio (Li), Magnésio (Mg), Manganês (Mn), Mercúrio (Hg), Níquel (Ni), Nitrato $\left(\mathrm{NO}_{3}\right)$, Ortofosfato, Potássio $(\mathrm{K})$, Prata $(\mathrm{Ag})$, Sódio $(\mathrm{Na})$, Sólidos Totais Dissolvidos (STD), Sulfato $\left(\mathrm{SO}_{4}\right)$, Zinco (Zn), Turbidez, Coliformes Totais e Escherichia coli.
A caracterização hidroquímica foi realizada com base na análise dos resultados de diferentes parâmetros e dos principais cátions e ânions.

\subsection{Avaliação da Qualidade de Água}

A avaliação da qualidade foi realizada a partir de três formas diferentes: comparação com VMPs, aplicação de índices de qualidade e avaliação de risco.

A primeira forma foi realizada por meio da comparação dos resultados dos diferentes parâmetros, obtidos com as análises feitas em campo e nas amostras coletadas, com os valores dos VMP's disponibilizados na Portaria 2914/2011 do Ministério da Saúde (Brasil, 2011). 
A segunda forma foi realizada com base na aplicação do Índice de Qualidade de Água Subterrânea (IQUAS), de Almeida \& Oliveira (2008).

A equação do IQUAS que foi utilizada para os cálculos é apresentada abaixo:

$$
\begin{aligned}
& \text { IQUAS }=I_{\mathrm{BIO}} 0,19 * I_{\mathrm{FEMN}} 0,06 * \mathrm{I}_{\mathrm{MS}} 0,19 * \mathrm{IPS}_{\mathrm{PS}} 0,06 * \mathrm{I}_{\mathrm{NIT}} \mathrm{O}, 19 * \mathrm{I}_{\mathrm{AMO}} 0,06 * \\
& \mathrm{I}_{\mathrm{MIN}} 0,06 * \mathrm{IORG}^{0,19}
\end{aligned}
$$

onde: $\mathrm{I}_{\mathrm{BIO}}=$ Índice de Microorganismos; $\mathrm{I}_{\mathrm{FEMN}}=$ Índice de Ferro e Manganês; I IMS = Índice de Mineralização e salinidade; IPS = Índice de Partículas em Suspensão; $I_{\mathrm{NIT}}=$ Índice de Nitrato; $I_{\mathrm{AMO}}=$ Índice de Nitrogenados; IMIN = Índice de Micropoluentes Minerais; IoRG = Índice de Micropoluentes Orgânicos.

Após a realização dos cálculos das equações, o resultado foi um número adimensional na faixa de 0 a 100 , que foi enquadrado em uma das cinco classes de qualidade da água, de acordo com a Figura 2.

Figura 2 - Faixas de classes de qualidade para a água subterrânea (0 a 100) para o índice de qualidade IQUAS

\begin{tabular}{|l|l|}
\hline $80-100=$ Ótima & \\
\hline $52-79=$ Boa & \\
\hline $37-51$ = Regular & \\
\hline $20-36=$ Ruim & \\
\hline 0 a $19=$ Péssima & \\
\hline
\end{tabular}

Fonte: Almeida \& Oliveira (2008)

Na segunda forma, foi aplicado o Índice Relativo de Risco (IRR), proposto por Athayde et al. (2014). Esse método se baseia no cálculo de um quociente $(\mathrm{Q})$ para cada parâmetro, realizado a partir da di- visão da concentração desse parâmetro (encontrado na análise) pelo seu Valor Máximo Permitido (VMP). 0 somatório dos quocientes de cada parâmetro determina o IRR da amostra, observado na Figura 3.

Figura 3 - Exemplo de cálculo do Índice Relativo de Risco (IRR)

\begin{tabular}{|c|c|c|c|}
\hline Amostra & Parâmetro $\mathrm{X}$ & Parâmetro $\mathrm{Y}$ & IRR \\
\hline $\mathrm{A}$ & Concentração c & Concentração g & valor \\
\hline
\end{tabular}

A terceira forma foi realizada por meio da Avaliação de Risco à Saúde Humana (USEPA, 1989), onde foi considerado um cenário residencial (rural e urbano) de ingestão de água subterrânea, cujos receptores escolhidos foram crianças (Tabela 2). A escolha das crianças como receptores foi adotada, pois é um cenário mais conservador, além do fato que há poços analisados nesse estudo que são utilizados no abastecimento de escolas de ensino fundamental. As equações utilizadas foram:

$$
\begin{gathered}
I=\mathrm{C} \times \frac{\mathrm{IR} \times \mathrm{EF} \times E D}{B W} \times \frac{1}{\mathrm{AT}} \\
\mathrm{HQ}=\frac{\mathrm{I}_{\mathrm{n}}}{\mathrm{RfD}_{\mathrm{i}}} \\
\mathrm{HI}=\Sigma \frac{\mathrm{I}_{\mathrm{n}}}{\mathrm{RfD}_{\mathrm{i}}}
\end{gathered}
$$

em que: I = Ingresso por ingestão de água contaminada (mg/kg.dia) $\mathrm{C}=$ Concentração do composto químico na água $(\mathrm{mg} / \mathrm{L}) ; \mathrm{IR}=$ Taxa de ingestão de água (L/dia); EF = Frequência da exposição (dias/ano); ED = Duração média da exposição (ano); BW = Peso corpóreo médio do individuo durante a exposição (kg); AT = Período de exposição médio durante a exposição (dias); $I_{n}=$ Dose de ingresso para o cenário de exposição "n" (mg/kg.dia); $\mathrm{RfD}_{\mathrm{i}}=$ Dose de referência para via de ingresso “i” (mg/kg.dia).

Tabela 2 - Parâmetros utilizados no cálculo do ingresso por ingestão de água contaminada

\begin{tabular}{llll}
\hline \multirow{2}{*}{ Parâmetro } & \multirow{2}{*}{ Fonte } & Residencial Rural \\
\cline { 3 - 4 } & & Adulto & Criança \\
\hline IR (L dia-1) & CETESB, 2009 & 350 & 1 \\
EF (dias ano-1) & CETESB, 2009 & 72 & 350 \\
ED (anos) & CETESB, 2001 & 63 & 6 \\
BW (kg) & COUTO, 2006 & 26280 & 15 \\
ATn (dias) & IBGE, 2008 & 26580 & 2190 \\
ATc (dias) & CETESB, 2009 & 26280 \\
\hline Obs: ATn (efeitos não carcinogênico); ATc (efeitos carcinogênicos)
\end{tabular}

\section{RESULTADOS}

\subsection{Hidrogeologia da Área de Estudo}

No município de Osório, o Sistema Aquífero Quaternário Costeiro I e II é caracterizado por aquíferos granulares livres e confinados, que ocorrem associados a camadas de sedimentos arenosos (Figura 1). No intervalo de 0 a $30 \mathrm{~m}$, há ocorrência de aquíferos granulares livres que ocorrem em camadas de areias fina, muito fina e média que estão intercaladas com camadas de argila ou argila arenosa.
Os aquíferos granulares livres são captados, principalmente, por poços ponteiras que possuem profundidades baixas (entre 15 e $20 \mathrm{~m}$ ) e pequenas vazões (entre 1,0 e 2,5 m³/h).

\subsection{Avaliação Hidroquímica da Água Subterrânea}

A caracterização hidroquímica foi realizada com base na análise de dados físico-químicos, que são apresentados nas Tabelas 3 e 4. 
Tabela 3 - Resultados dos principais parâmetros e constituintes principais da água subterrânea

\begin{tabular}{|c|c|c|c|c|c|c|c|c|c|c|c|}
\hline Amostra & $\mathrm{pH}$ & $\begin{array}{c}\text { CE } \\
(\omega S / \mathrm{cm})\end{array}$ & $\begin{array}{l}\text { STD } \\
(\mathrm{mg} / \mathrm{l})\end{array}$ & $\begin{array}{c}\text { Alcalinidade } \\
(\mathrm{mg} / \mathrm{l})\end{array}$ & $\begin{array}{c}\text { Dureza } \\
(\mathrm{mg} / \mathrm{l})\end{array}$ & $\begin{array}{c}\mathrm{Na} \\
(\mathrm{mg} / \mathrm{I})\end{array}$ & $\begin{array}{c}\mathrm{K} \\
(\mathrm{mg} / \mathrm{I})\end{array}$ & $\begin{array}{c}\mathrm{Ca} \\
(\mathrm{mg} / \mathrm{l})\end{array}$ & $\begin{array}{c}\mathrm{Mg} \\
(\mathrm{mg} / \mathrm{l})\end{array}$ & $\begin{array}{c}\mathrm{Cl} \\
(\mathrm{mg} / \mathrm{l})\end{array}$ & $\begin{array}{c}\mathrm{SO}_{4} \\
(\mathrm{mg} / \mathrm{l})\end{array}$ \\
\hline PP01 & 5,6 & 49,7 & 57,0 & 5,0 & 8,0 & 5,17 & 0,98 & 0,86 & 1,22 & 7,5 & ND \\
\hline PP02 & 5,1 & 49,9 & 59,0 & 8,0 & 7,0 & 4,51 & 0,97 & 0,51 & 1,03 & 8,5 & ND \\
\hline PP03 & 6,2 & 107,4 & 170,0 & 40,0 & 25,0 & 11,30 & 2,32 & 4,99 & 1,24 & 7,5 & 2,85 \\
\hline PP04 & 7,1 & 510,2 & 358,0 & 160,0 & 190,0 & 28,30 & 0,48 & 35,30 & 4,17 & 60,0 & 23,50 \\
\hline PP06 & 6,5 & 137,5 & 159,0 & 45,0 & 60,0 & 4,04 & 3,44 & 13,9 & 2,69 & 7,5 & ND \\
\hline PP07 & 6,2 & 188,4 & 169,0 & 35,0 & 55,0 & 11,60 & 4,89 & 10,20 & 3,31 & 22,5 & 8,52 \\
\hline PP08 & 7,6 & 462,1 & 240,0 & 185,0 & 150,0 & 31,60 & 10,20 & 30,10 & 13,2 & 30 & ND \\
\hline PP09 & 7,4 & 329,4 & 194,0 & 140,0 & 90,0 & 24,10 & 11,40 & 14,30 & 12,9 & 20 & ND \\
\hline PP10 & 5,1 & 52,6 & 54,0 & 10,0 & 10,0 & 5,43 & 0,49 & 0,60 & 1,01 & 10 & ND \\
\hline PP13 & 5,7 & 59,6 & 55,0 & 15,0 & 10,0 & 6,01 & 1,40 & 1,70 & 0,58 & 7,5 & 1,54 \\
\hline PP14 & 5,6 & 98,7 & 126,0 & 40,0 & 60,0 & 1,95 & 0,31 & 5,84 & 0,82 & 13,5 & 5.26 \\
\hline PP16 & 6,5 & 55,3 & 47,0 & 15,0 & 10,0 & 1,96 & 0,47 & 0,61 & 0,06 & 7,5 & ND \\
\hline PP17 & 5,7 & 75,3 & 73,0 & 10,0 & 12,0 & 0,96 & 0,20 & 0,39 & 0,16 & 7,5 & 2,59 \\
\hline PP19 & 4,8 & 111,6 & 116,0 & 5,0 & 25,0 & 3,34 & 0,37 & 1,78 & 3,11 & 7,5 & 8,98 \\
\hline PP20 & 5 & 87 & 120,0 & 10,0 & 30,0 & 1,99 & 1,53 & 4,08 & 1,95 & 5 & 5,85 \\
\hline
\end{tabular}

Tabela 4 - Resultados para metais, nitrato e fluoreto da água subterrânea

\begin{tabular}{|c|c|c|c|c|c|c|c|c|c|c|c|}
\hline Amostra & $\begin{array}{c}\mathrm{Al} \\
(\mathrm{mg} / \mathrm{l})\end{array}$ & $\begin{array}{c}\mathrm{Pb} \\
(\mathrm{mg} / \mathrm{l})\end{array}$ & $\begin{array}{c}\mathrm{Cu} \\
(\mathrm{mg} / \mathrm{l})\end{array}$ & $\begin{array}{c}\mathrm{Cr} \\
(\mathrm{mg} / \mathrm{l})\end{array}$ & $\begin{array}{c}\mathrm{Fe} \\
(\mathrm{mg} / \mathrm{I})\end{array}$ & $\begin{array}{c}\mathrm{Mn} \\
(\mathrm{mg} / \mathrm{l})\end{array}$ & $\begin{array}{c}\mathrm{Hg} \\
(\mathrm{mg} / \mathrm{l})\end{array}$ & $\begin{array}{c}\mathrm{Ni} \\
(\mathrm{mg} / \mathrm{l})\end{array}$ & $\begin{array}{c}\mathrm{Zn} \\
(\mathrm{mg} / \mathrm{l})\end{array}$ & $\begin{array}{c}\mathrm{NO}_{3} \\
(\mathrm{mg} / \mathrm{l})\end{array}$ & $\begin{array}{c}F \\
(\mathrm{mg} / \mathrm{l})\end{array}$ \\
\hline PP01 & ND & ND & ND & ND & ND & ND & 0,0004 & ND & ND & 1,45 & ND \\
\hline PP02 & ND & ND & ND & ND & ND & ND & 0,0005 & ND & ND & 0,89 & ND \\
\hline PP03 & ND & ND & 0,013 & ND & 9,910 & ND & ND & 0,0006 & ND & 0,39 & ND \\
\hline PP04 & ND & ND & ND & 0,002 & 3,660 & 0,745 & 0,0007 & ND & ND & 0,64 & 0,18 \\
\hline PP06 & ND & ND & ND & ND & 0,058 & ND & ND & ND & ND & 2,98 & ND \\
\hline PP07 & ND & ND & ND & ND & 0,229 & ND & ND & ND & ND & 1,60 & ND \\
\hline PP08 & ND & 0,005 & 0,052 & ND & 0,438 & ND & ND & ND & 0,016 & 0,33 & 0,36 \\
\hline PP09 & 0,050 & ND & ND & ND & 0,117 & ND & ND & ND & ND & ND & 0,32 \\
\hline PP10 & ND & ND & 0,005 & ND & 0,089 & ND & 0,0006 & ND & ND & 0,31 & ND \\
\hline PP13 & ND & ND & ND & ND & 0,077 & ND & 0,0009 & ND & ND & 0,51 & ND \\
\hline PP14 & 0,169 & ND & ND & ND & 0,116 & ND & 0,0006 & ND & ND & 1,16 & ND \\
\hline PP16 & ND & ND & ND & ND & ND & ND & 0,0007 & ND & ND & 0,29 & ND \\
\hline PP17 & ND & ND & ND & ND & ND & ND & ND & ND & ND & 1,23 & ND \\
\hline PP19 & 0,317 & ND & ND & ND & ND & 0,203 & ND & ND & ND & 3,84 & ND \\
\hline PP20 & 0,201 & ND & ND & ND & ND & ND & ND & ND & ND & 6,19 & ND \\
\hline
\end{tabular}

A partir da análise dos dados obtidos, é possível observar que os valores de $\mathrm{pH}$ e condutividade elétrica (CE) foram variáveis (entre $4,6$ e 7,6, sendo que $77 \%$ apresentam valores inferiores a 6,5$)$, indicando a ocorrência de águas ácidas a levemente ácidas. A condutividade elétrica (CE), apresentou valores entre 40 a $500 \mathrm{uS} / \mathrm{cm}$, sendo que a maior parte das águas (84\%), possui valores inferiores a $200 \mathrm{uS} / \mathrm{cm}$, indicando uma baixa mineralização. Com relação aos sólidos totais dissolvidos (STD), 91\% das amostras apresentaram valores inferiores a $200 \mathrm{mg} / \mathrm{l}$.

Com base na análise dos principais cátions e ânions pode-se observar que as águas apresentam diferentes composições. A maior parte das amostras apresentam águas com baixas concentrações para os principais cátions e ânions, valores de pH e CE baixos. Essas características podem estar relacionadas com pouco tempo de residência, visto que a concentração de elementos é baixa, assim como os valores de pH e CE. Por outro lado, há ocorrência de amostras de água que apresentam valores mais elevados para cátions e ânions, bem como para os parâmetros pH e CE. Essas águas apresentam valores maiores de alcalinidade, $\mathrm{pH}$ (superior a 7,0) e CE (maiores entre 329 e 511 us/cm), sendo encontradas em poços que apresentam maior profundidade.

\subsection{Avaliação da Qualidade da Água Subterrânea}

\subsubsection{Comparação com os Valores Máximos Permitidos (VMPs)}

Os resultados da análise da qualidade da água captada, realizada via comparação dos valores encontrados para os diferentes parâmetros, com os valores máximos permitidos (VMP da portaria 2914/11 do Ministério da Saúde), são apresentados na Tabela 5. 
Tabela 5 - Comparação dos parâmetros analisados com os Valores Máximos Permitidos (VMP's) pela Portaria 2914/11 do Ministério da Saúde (MS) nas amostras analisadas no laboratório

\begin{tabular}{|c|c|c|c|c|c|c|c|c|c|}
\hline Amostras & $\mathrm{Al}$ & $\mathrm{NH}_{3}$ & Cor & $\mathrm{Fe}$ & $\mathrm{Mn}$ & $\mathrm{pH}$ & Turbidez & C. Totais & E. coli \\
\hline PP01 & ND & 0,5 & 5,0 & ND & ND & 5,6 & ND & $<1$ & $<1$ \\
\hline PP02 & ND & 0,6 & 5,0 & ND & ND & 5,1 & ND & $<1$ & $<1$ \\
\hline PP03 & ND & 0,8 & 75,0 & 9,910 & ND & 6,2 & 18,7 & $<1$ & $<1$ \\
\hline PP04 & ND & 0,9 & 50,0 & 3,660 & 0,745 & 7,1 & 29,4 & $<1$ & $<1$ \\
\hline PP06 & ND & 1,5 & 10,0 & 0,058 & ND & 6,5 & 2,57 & 121,0 & 86,0 \\
\hline PP07 & ND & 1,8 & 5,0 & 0,229 & ND & 6,2 & 1,84 & 335,0 & 175,0 \\
\hline PP08 & ND & 4 & 10,0 & 0,438 & ND & 7,6 & 3,9 & $<1$ & $<1$ \\
\hline PP09 & 0,050 & 3,6 & 5,0 & 0,117 & ND & 7,4 & ND & $<1$ & $<1$ \\
\hline PP10 & ND & 0,3 & 5,0 & 0,089 & ND & 5,1 & ND & $<1$ & $<1$ \\
\hline PP13 & ND & 0,4 & 5,0 & 0,077 & ND & 5,7 & ND & $<1$ & $<1$ \\
\hline PP14 & 0,169 & 0,3 & 5,0 & 0,116 & ND & 5,6 & 7,27 & 2014,0 & 504,0 \\
\hline PP16 & ND & 0,4 & 5,0 & ND & ND & 6,5 & ND & $<1$ & $<1$ \\
\hline PP17 & ND & 0,5 & 5,0 & ND & ND & 5,7 & ND & $<1$ & $<1$ \\
\hline PP19 & 0,317 & 0,3 & 5,0 & ND & 0,203 & 4,8 & ND & $<1$ & $<1$ \\
\hline PP20 & 0,201 & 0,6 & 5,0 & ND & ND & 5,0 & ND & 5794,0 & 495,0 \\
\hline VMP & $\begin{array}{c}0,2 \\
\mathrm{mg} / \mathrm{L}\end{array}$ & $\begin{array}{c}1,5 \\
\mathrm{mg} / \mathrm{L}\end{array}$ & $15 \mathrm{uH}$ & $\begin{array}{c}0,3 \\
\mathrm{mg} / \mathrm{L}\end{array}$ & $\begin{array}{c}0,1 \\
\mathrm{mg} / \mathrm{L}\end{array}$ & $\begin{array}{c}6,0 \text { a } \\
9,5\end{array}$ & 5 uT & $\begin{array}{c}\text { Ausente em } \\
100 \mathrm{ml} \\
(\mathrm{NMP} / 100 \mathrm{ml})\end{array}$ & $\begin{array}{c}\text { Ausente em } \\
100 \mathrm{ml} \\
\text { (NMP/100ml) }\end{array}$ \\
\hline \multicolumn{3}{|c|}{ ND - Não Detectado } & \multicolumn{6}{|c|}{ Coloração vermelha - parâmetros acima do valor máximo permitido } & \\
\hline Al = Alumínio & \multicolumn{2}{|c|}{$\mathrm{NH}_{3}=$ Amônia } & \multicolumn{2}{|c|}{$\mathrm{Fe}=$ Ferro } & $M n=M$ & anês & \multicolumn{2}{|c|}{ C.Totais $=$ Coliformes Totais } & \\
\hline
\end{tabular}

Os resultados indicam que o parâmetro $\mathrm{pH}$ é o que mais aparece fora do limite (entre 6,0 e 9,5), estando abaixo de 6,0 em 8 poços (PP01, PP02, PP10, PP13, PP14, PP17, PP19, PP20). A ocorrência de coliformes foi identificada em 4 amostras, com valores entre 86 e 5794 NMP/100ml (PP06, PP07, PP14 e PP20). Os outros parâmetros que apresentaram valores acima dos VMPs foram alumínio (poços PP19 e PP20), amônia (poços PP07, PP08 e PP09), cor (poços PP03 e PP04), ferro (poços PP03, PP04 e PP08), manganês (poços PP04 e PP19) e turbidez (poços PP03, PP04 e PP14). Os outros parâmetros analisados como chumbo, cloreto, cobre, cromo, dureza, fluoreto, mercúrio, níquel, nitrato, sódio, sólidos totais dissolvidos, sulfato e zinco, estão todos dentro dos limites permissíveis, aparecendo em pequenas concentrações ou não sendo detectados em algumas amostras.
Essa avaliação indicou que dos 15 poços analisados, 14 apresentaram algum parâmetro acima dos VMPs. Se não for levado em conta os valores de $\mathrm{pH}$, ainda haverá uma grande ocorrência de poços (9 amostras), apresentando um ou mais parâmetros acima dos VMPs.

\subsection{2. Índice de Qualidade da Água Subterrânea (IQUAS)}

Para aplicação do IQUAS foram utilizados os dados apresentados nas Tabelas 3 e 4, para cálculo dos valores dos grupos de alteração e do índice (equação 1). Os resultados são apresentados na Tabela 6.

Tabela 6 - Valores utilizados para a determinação da Qualidade da Água, segundo o índice de Qualidade da Água Subterrânea (IQUAS) e seu resultado de acordo com as faixas de classes de qualidade para a água subterrânea (0 a 100)

\begin{tabular}{|c|c|c|c|c|c|c|c|c|c|}
\hline Amostra & IBIO & IFEMN & IMS & IPS & INIT & IAMO & IMIN & IORG & IQUAS \\
\hline PP01 & 100 & 100 & 77,04 & 100 & 100 & 66,61 & 52,00 & 100 & 89 \\
\hline PP02 & 100 & 100 & 116,69 & 100 & 100 & 63,85 & 51,25 & 100 & 96 \\
\hline PP03 & 100 & 36,33 & 77,14 & 22,97 & 100 & 59,72 & 100 & 100 & 79 \\
\hline PP04 & 100 & 22,70 & 72,70 & 27,50 & 100 & 58,10 & 48,54 & 100 & 74 \\
\hline PP06 & 0 & - & - & - & - & - & - & - & 0 \\
\hline PP07 & 0 & - & - & - & - & - & - & - & 0 \\
\hline PP08 & 100 & 70,61 & 81,32 & 44,11 & 100 & 41,08 & 100 & 100 & 85 \\
\hline PP09 & 100 & 93,55 & 83,45 & 100 & 100 & 42,10 & 100 & 100 & 91 \\
\hline PP10 & 100 & 100 & 73,21 & 100 & 100 & 75,01 & 50,37 & 100 & 90 \\
\hline PP13 & 100 & 100 & 77,68 & 100 & 100 & 70,15 & 47,11 & 100 & 89 \\
\hline PP14 & 0 & - & - & - & - & - & - & - & 0 \\
\hline PP16 & 100 & 100 & 81,86 & 100 & 100 & 70,15 & 48,92 & 100 & 90 \\
\hline PP17 & 100 & 100 & 76,94 & 100 & 100 & 66,61 & 100 & 100 & 93 \\
\hline PP19 & 100 & 64,72 & 72,37 & 100 & 74,12 & 75,01 & 100 & 100 & 85 \\
\hline PP20 & 0 & - & - & - & - & - & - & - & 0 \\
\hline
\end{tabular}

A partir destes cálculos foi feita a classificação das águas, onde 9 amostras foram classificadas como ótimas (PP01, PP02, PP08, PP09, PP10, PP13, PP16, PP17 e PP19), 4 amostras foram péssimas (PP06, PP07, PP14 e PP20) e 2 amostras foram boas (PP03 e PP04).
Deve-se destacar que os poços que apresentaram águas classificadas como péssimas são os que possuem presença de Coliformes Totais e E.coli. Como a presença já indica uma qualidade péssima, os outros parâmetros acabam não sendo avaliados. 
As amostras classificadas como boas apresentam alterações acima dos VMPS para os seguintes parâmetros: cor, ferro, manganês e turbidez.

\subsubsection{Cálculo do Índice Relativo de Risco (IRR)}

O IRR foi calculado a fim de hierarquizar as amostras conforme os valores encontrados para observar quais possuem maiores ou menores valores de risco. Esse índice não indica diretamente os problemas de qualidade de água, mas pode ser utilizado para avaliar quais amostras apresentam maiores riscos, visto que valores mais elevados de IRR, são obtidos quando há ocorrência de parâmetros com concentrações elevadas, muitas vezes, superiores aos VMP's.

Os elementos usados (Tabelas 3 e 4) foram $\mathrm{Al}, \mathrm{Pb}, \mathrm{Cl}, \mathrm{Cu}, \mathrm{Cr}, \mathrm{Fe}, \mathrm{F}$, $\mathrm{Mn}, \mathrm{Hg}, \mathrm{Ni}, \mathrm{NO}_{3}, \mathrm{Na}, \mathrm{SO}_{4}$ e $\mathrm{Zn}$ e os parâmetros dureza e STD. $\mathrm{Na}$ Tabela 7 é apresentado o valor do IRR para cada amostra.

Tabela 7 - Valor do Índice Relativo de Risco (IRR) calculado para cada amostra

\begin{tabular}{|c|c|c|c|c|c|c|c|c|c|c|c|c|c|c|c|}
\hline & PP & PP & PP & PP & PP & PP & PP & PP & PP & PP & PP & PP & PP & PP & PP \\
\hline & 01 & 02 & 03 & 04 & 06 & 07 & 08 & 09 & 10 & 13 & 14 & 16 & 17 & 19 & 20 \\
\hline IRR & 1,35 & 1,34 & 34,08 & 22,19 & 1,51 & 2,02 & 3,46 & 1,85 & 1,55 & 1,89 & 5,99 & 1,47 & 1,08 & 4,78 & 2,45 \\
\hline
\end{tabular}

De acordo com o IRR, pode-se observar que as amostras PP03 e PP04 apresentaram valores muito elevados de IRR $(34,084$ e 22,198, respectivamente). Outras cinco amostras (PP07, PP08, PP14, PP19 e PP20) apresentaram valores entre 2 e 6 de IRR). A amostra PP03, classificada como boa pelo IQUAS, tem presença de ferro, níquel e sódio. A amostra PP04, também classificada como boa pelo IQUAS, tem presença de ferro, cromo, manganês, sódio e pequenas concentrações de mercúrio, cobre e zinco. A amostra PP14 tem presença de alumínio e coliformes, enquanto que a amostra PP19, classificada como ótima, possui presença de alumínio e manganês.

\subsubsection{Avaliação de Risco à Saúde}

Para a avaliação de risco a saúde, foi calculado a dose de ingresso (I) e o quociente de perigo (HQ) para cada elemento, na situação de efeito não carcinogênico (equações 2,3 e 4). Foram selecionados os elementos alumínio, cobre, chumbo, ferro, manganês, zinco, fluoretos e nitrato (Tabela 4), pois esses possuem informações sobre as doses de referência oral ou de ingestão, na planilha da CETESB (2013). 0 cenário escolhido foi o residencial rural para crianças. $O$ índice de perigo não carcinogênico $(\mathrm{HI})$ foi calculado a partir do somatório dos HQs, dos elementos de cada poço (Tabela 7).

Tabela 7 - Valores de HQ e HI calculados para a Avaliação de Risco à Saúde Humana

\begin{tabular}{cccccccccc}
\hline Amostra & $\mathrm{HQ}(\mathrm{Al})$ & $\mathrm{HQ}(\mathrm{Fe})$ & $\mathrm{HQ}(\mathrm{F})$ & $\mathrm{HQ}(\mathrm{Mn})$ & $\mathrm{HQ}\left(\mathrm{NO}_{3}\right)$ & $\mathrm{HQ}(\mathrm{Zn})$ & $\mathrm{HQ}(\mathrm{Cu})$ & $\mathrm{HQ}(\mathrm{Pb})$ & $\mathrm{HI}$ \\
\hline PP01 & 0,0030 & 0,0032 & 0,0900 & 0,0027 & 0,0578 & 0,00253 & 0,00027 & 0,0244 & 0,1839 \\
PP02 & 0,0030 & 0,0032 & 0,0900 & 0,0027 & 0,0355 & 0,00253 & 0,00027 & 0,0244 & 0,1616 \\
PP03 & 0,0030 & 0,9040 & 0,0900 & 0,0027 & 0,0158 & 0,00253 & 0,02075 & 0,0244 \\
PP04 & 0,0030 & 0,3340 & 0,2875 & 0,3400 & 0,0253 & 0,00253 & 0,00027 & 0,0244 & 1,0170 \\
PP06 & 0,0030 & 0,0052 & 0,0900 & 0,0027 & 0,1190 & 0,00253 & 0,00027 & 0,0244 & 0,2471 \\
PP07 & 0,0030 & 0,0208 & 0,0900 & 0,0027 & 0,0638 & 0,00253 & 0,00027 & 0,0244 & 0,2075 \\
PP08 & 0,0030 & 0,0398 & 0,3300 & 0,0027 & 0,0130 & 0,00340 & 0,08250 & 0,0852 \\
PP09 & 0,0031 & 0,0105 & 0,5500 & 0,0027 & 0,0079 & 0,00253 & 0,00027 & 0,0244 \\
PP10 & 0,0030 & 0,0080 & 0,0900 & 0,0027 & 0,0122 & 0,00253 & 0,00797 & 0,0244 & 0,6014 \\
PP13 & 0,0030 & 0,0070 & 0,0900 & 0,0027 & 0,0205 & 0,00253 & 0,00027 & 0,0244 & 0,1504 \\
PP14 & 0,0107 & 0,0108 & 0,0900 & 0,0027 & 0,0463 & 0,00253 & 0,00027 & 0,0244 & 0,1877 \\
PP16 & 0,0030 & 0,0032 & 0,0900 & 0,0027 & 0,0117 & 0,00253 & 0,00027 & 0,0244 & 0,1378 \\
PP17 & 0,0030 & 0,0032 & 0,0900 & 0,0027 & 0,0490 & 0,00253 & 0,00027 & 0,0244 & 0,1751 \\
PP19 & 0,0202 & 0,0032 & 0,0900 & 0,0921 & 0,1533 & 0,00253 & 0,00027 & 0,0244 & 0,3860 \\
PP20 & 0,0128 & 0,0032 & 0,0900 & 0,0027 & 0,2471 & 0,00253 & 0,00027 & 0,0244 & 0,3830 \\
\hline
\end{tabular}

Conforme a Tabela 7, pode-se observar que as amostras que apresentam um risco não carcinogênico (HI acima de 1), correspondem aos poços PP03 e PP04, que apresentam concentrações maiores para os parâmetros ferro e manganês. As amostras PP09 (presença de chumbo, cobre, ferro, zinco, fluoretos e nitrato) e PP08 (presença de alumínio, ferro e fluoretos) possuem um $\mathrm{HI}$ alto, mas não apresentam risco não carcinogênico.

\subsection{Análise Comparativa dos Resultados dos Diferentes Índices de Qualidade de Água Subterrânea}

Após serem calculados o Índice de Qualidade da Água Subterrânea (IQUAS), o Índice Relativo de Risco (IRR) e o Índice de Perigo Não Carcinogênico $(\mathrm{HI})$, foi feita uma comparação e avaliação entre eles. Os resultados dos índices também foram comparados com os Valores Máximos Permitidos (VMP's), definidos na Portaria 2914/2011 do Ministério da Saúde, apresentados na Tabela 8. 
Tabela 8 - Comparativo entre os métodos utilizados

\begin{tabular}{ccccc}
\hline Amostra & IQUAS & IRR & HI & Acima dos VMP's \\
\hline PP01 & 89 Ótimo & 1,352 & 0,1839005 & $\mathrm{pH}$ \\
PP02 & 96 Ótimo & 1,348 & 0,1616005 & FH \\
PP08 & 85 Ótimo & 3,548 & 0,5596 & Amônia \\
PP09 & 91 Ótimo & 1,852 & 0,6014005 & $\mathrm{pH}$ \\
PP10 & 90 Ótimo & 1,546 & 0,150808 & $\mathrm{pH}$ \\
PP13 & 89 Ótimo & 1,887 & 0,1504005 & - \\
PP16 & 90 Ótimo & 1,473 & 0,1378005 & $\mathrm{pH}$ \\
PP17 & 93 Ótimo & 1,081 & 0,1751005 & Alumínio; Manganês; pH \\
PP19 & 85 Ótimo & 4,777 & 0,3860005 & Cor; Ferro; Turbidez \\
PP03 & 79 Bom & 34,084 & 1,063183 & Cor; Ferro; Manganês; Turbidez \\
PP04 & 74 Bom & 22,198 & 1,0170005 & Coliformes; E.coli \\
PP06 & 0 Péssimo & 1,515 & 0,2471005 & Ferro; Coliformes; E.coli; Amônia \\
PP07 & 0 Péssimo & 2,022 & 0,2075005 & Turbidez; Coliformes; E.col;; pH \\
PP14 & 0 Péssimo & 5,995 & 0,1877005 & Coliformes; E.col;; Alumínio; pH \\
PP20 & 0 Péssimo & 2,447 & 0,3830005 & \\
\hline
\end{tabular}

Analisando os resultados pode-se observar que os valores do IQUAS mostram que amostras que apresentam boa ou ótima qualidade podem ter problemas que não são detectados. As amostras PP01, PP02, PP10, PP13 e PP17 têm problemas no pH, enquanto as amostras PP08 e PP09 têm problemas com amônia e ferro. Outras amostras como a PP03, PP04 e PP19 apresentam problemas com ferro, alumínio, manganês, entre outros parâmetros.

Já as amostras que tiveram ocorrência de coliformes fecais e E. Coli foram classificadas pelo IQUAS como péssimas. A amostra PP06 só teve esse parâmetro alterado, enquanto as outras amostras PP07, PP14 e PP20, apresentaram problemas em outros parâmetros (ferro, amônia, turbidez, alumínio). Isso tem importância, pois as medidas que podem ser tomadas para reverter o problema bacteriológico são mais fáceis de serem implantadas e executadas, do que medidas necessárias para a remoção dos metais.

O Índice Relativo de Risco (IRR) apresentou-se como uma ferramenta interessante para avaliação de amostras que não apresentam um risco eminente, mas que podem vir a ter problemas futuros, como as amostras PP08 e PP19, que possuem um IQUAS ótimo, mas um IRR alto.

O índice de perigo não carcinogênico indicou a ocorrência de risco nas amostras PPO3 e PPO4 que, de acordo com o IQUAS, foram classificadas como boas. Essas amostras apresentaram os maiores valores de IRR, indicando que elas possuem parâmetros com elevada concentração, fato observado quando da comparação com os VMP's, que indicaram valores de cor, ferro, manganês e turbidez acima dos limites.

A única amostra que teve um comportamento regular foi a PP16, com IQUAS ótimo, baixo valor de IRR e HI, além de não possuir nenhum parâmetro acima dos limites permitidos. As amostras PP01, PP02, PP10, PP13 e PP17 apresentaram IQUAS ótimo, valores baixos de IRR e HI, e apenas apresentaram valores fora dos limites de $\mathrm{pH}$, que podem ser facilmente ajustáveis.

Os métodos utilizados para avaliação da qualidade apresentaram um bom desempenho. O Índice da Qualidade da Água Subterrânea (IQUAS) foi o que demonstrou uma menor representatividade da si- tuação das águas, pois algumas amostras classificadas como ótimas, quando comparadas com os Valores Máximos Permitidos, apresentaram falhas de qualidade. 0 Índice Relativo de Risco demonstrou ser uma boa ferramenta para avaliação de problemas futuros, pois permite identificar as amostras que apresentam maiores índices, obtidos pela concentração de elementos químicos. Já a Avaliação do Risco a Saúde Humana, via ingestão de água subterrânea, tem importância para a identificação de amostras que apresentam risco não carcinogênico.

\section{CONCLUSÕES}

Na área de estudo, abrangida pelo município de Osório/RS, há ocorrência do Sistema Aquífero Quaternário Costeiro I e II (SAQC), que é caracterizado por aquíferos granulares livres e confinados. 0 aquífero granular livre é o de maior importância para a população, que capta água através de poços ponteiras, para uso no abastecimento. As águas subterrâneas do aquífero livre são caracterizadas por baixa concentração de cátions e ânions e baixos valores de $\mathrm{pH}$ e condutividade elétrica.

Os resultados da avaliação da qualidade da água demonstraram a ocorrência de problemas de qualidade. Na comparação feita com os VMP's, foram observados problemas em 14 poços, associados aos parâmetros $\mathrm{pH}$, amônia, ferro, cor, manganês, turbidez, Coliformes Totais e E.coli. Com o uso do IQUAS, foram identificados 4 poços que apresentaram águas classificadas como péssimas. 0 cálculo do IRR indicou a ocorrência de 7 amostras com valores altos PP03 (34,084), PP04 (22,198), PP14 (5,995), PP19 (4,777), PP08 $(3,548)$, PP20 $(2,447)$ e PP07 $(2,022)$, sendo que todas tiveram pelo menos um parâmetro acima dos VMP's. Por fim, a Avaliação de Risco a Saúde, para ingestão da água subterrânea, resultou na identificação de 2 amostras que apresentaram Risco não Carcinogênico (poços PP03 e PP04).

A partir deste trabalho pode-se verificar que a aplicação em conjunto, de diferentes índices de avaliação da qualidade da água subterrânea, tem grande importância, pois com os mesmos é possível avaliar melhor os problemas de qualidade de água de uma determinada região. 


\section{AGRADECIMENTOS}

Ao Projeto Lagoas Costeiras 3 (LACOSIII / UCS).

\section{REFERÊNCIAS}

ALMEIDA, R. A. S; OLIVEIRA, I. B. Aplicação do Índice de Qualidade de Água Subterrânea (IQUAS) destinado ao uso para Consumo Humano. In: CONGRESSO BRASILEIRO DE ÁGUAS SUBTERRÂNEAS, 15., 2008. Anais... Natal, RN, 2008.

ALMEIDA, R. A. S. Índice de qualidade de uso da água subterrânea (e-IQUAS): uma metodologia de modelagem numérica flexível. Tese (Doutorado) - Universidade Federal da Bahia, Escola Politécnica. Salvador, BA, 2012.

ALMEIDA, R. A. S; OLIVEIRA, I. B. Índice de Qualidade de Uso da Água Subterrânea (e-iquas): aplicação para comunicar o estado da água em dois estudos de caso - Camaçari (BA) e Verdelândia (MG). Revista Águas Subterrâneas, v. 31 n. 1, p. 88- 103, 2017.

ATHAYDE. C. M.; ATHAYDE, G.B.; LICHT, O.A.B.; ROSA FILHO, E.F.DA. Índice Relativo de Risco, ferramenta de tomada de decisão em bacias hidrográficas destinadas ao abastecimento público. Boletim Paranaense de Geociências, v. 71 , n. 1 , p. $21-36,2014$

BATISTA, D.F.; CABRAL, J.B.P. Modelos matemáticos para avaliação do índice de qualidade da água: uma revisão. Acta Geográfica Boa Vista. v. 11, n. 25 , p. 111-136, jan/abr de 2017.

BRASIL. Ministério da Saúde. Portaria MS n².914/2011. Brasília. p.34. Disponívelem:<http://bvsms.saude.gov.br/bvs/saudele-

gis/gm/2011/prt2914_12_12_2011.html.> Acesso em 16 abril, 2018.

CADILHAC, L.; ALBINET, M. (Coord.). SEQ - Système d'évaluation de la qualité des eaux souterraines: Rapport de présentation. Agences de l'Eau. 2003. $75 p$

COUTINHO, J.V.; ALMEIDA, C.N.; GADELHA, C.L.M.; TARGINO, D.F.; LINHARES F.M.; COELHO, V.H.R. Avaliação Integrada da Qualidade da Água Subterrânea em uma Bacia Hidrográfica Representativa do Litoral da Região Nordeste do Brasil. RBRH - Revista Brasileira de Recursos Hídricos, v. 18, n. 4 , p. 197-212, out./dez. 2013.

FERNANDES, R.A.; LOUREIRO, C.O. Índice relativo de qualidade (irq): um método para caracterização e hierarquização do potencial qualitativo das águas subterrâneas. In: CONGRESSO BRASILEIRO DE ÁGUA SUBTERRÂNEA, 14., 2006, Curitiba. Anais... Curitiba: ABAS, 2006. 1 CD-ROM

FERREIRA, L.M.; IDE, C.N. 2001. Avaliação comparativa da sensibilidade do IQA-NSF, IQA-Smith e IQA-Horton, aplicados ao Rio Miranda, MS. In: CONGRESSO BRASILEIRO DE ENGENHARIA SANITÁRIA E AMBIENTAL, 2001, João Pessoa. Anais... João Pessoa: ABES, 2001. Disponível em:<http://www.bvs.br/php/index.php>. Acesso em: 28 nov. 2015.

FRANCA, R. M.; GOMES, M. Da C.R.; DA SILVA, F.J.A.; LIMA, M.G. De S. Análise multivariada dos parâmetros de qualidade das águas subterrâneas em Juazeiro do Norte - CE (Brasil). Águas Subterrâneas, v. 32, n. 1, p. 106-113, 2018.

MACHADO, J. L. F.; FREITAS, M. A. de. Projeto Mapa Hidrogeológico do Rio Grande do Sul: relatório final. Porto Alegre. CPRM. 65p. il. mapa. 2005.

OLIVEIRA, I. B., NEGRÃO, F. I, e SILVA, A. G. L.S. Mapeamento dos Aquíferos do Estado da Bahia utilizando o Índice de Qualidade Natural das Águas Subterrâneas - IQNAS. Revista Científica Água Subterrânea, v .21, n.. 1, p. 123137, 2007.

REGINATO, P.A.R.; MICHALSKI, E.Z.; PAIM, R.A. Água Subterrânea na Região de Osório. In: SCHÄFER, A.; LANZER. R.; SCUR, L. Atlas Socioambiental do Município de Osório. Caxias do Sul: EDUCS, 2017.

SÁNCHEZ, E.; COLMENAREJO, M.F.; VICENTE, J., RUBIO, A.; GARCÍA, M.G., TRAVIESO, L.; BORJA, R. Use of the Water Quality Index and Dissolved Oxygen Deficit as Simple Indicators of Watersheds Pollution. Ecological Indicators, v. 7 , n. 2 , p. $315-328.2007$

SANTOS, J, M, M. Índice de qualidade de água subterrânea aplicado em área de aquíferos cristalinos como uso agrícola: Bacia do Rio São Domingos-RJ. 2009, 189f. Tese (Doutorado em Geologia) - Universidade Federal do Rio de Janeiro, Rio de Janeiro.

STIGTER, T.Y.; RIBEIRO, L.; CARVALHO DILL, .A.M.M. Application of a Ground water Quality Index as an Assessment and Communication Tool In Agro-Environmental Policies - Two Portuguese Case Studies. Journal of Hydrology, 327, p. $578-591,2006$

USEPA, 1989. Risk Assessment Guidance for Superfund - Volume I - Human Health. Evaluation Manual (Part A). Disponível em: <http://www.epa.gov/oswer/riskassessment/ragsa/pdf/rags_a.pdf>. Acesso em: 10 março 2018. 